\title{
A comparison of three different surgical procedures in the treatment of type A thoracolumbar fractures: a randomized controlled trial
}

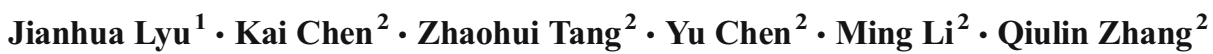

Received: 23 December 2015 / Accepted: 8 February 2016 / Published online: 24 February 2016

(C) The Author(s) 2016. This article is published with open access at Springerlink.com

\begin{abstract}
Purpose The aim of the study was to evaluate the efficacy of three different surgical procedures in the treatment of type A thoracolumbar fractures.

Materials and methods Between September 2012 and January 2015, a total of 90 patients with type A thoracolumbar fractures were randomly assigned into three groups of 30 each. Patients in group A, B, and C were treated with threelevel percutaneous fixation, two-level percutaneous fixation, and three-level open fixation, respectively. Blood loss, duration of surgery, VAS scores, Cobb angles, and anterior height ratios of fractured vertebrae were collected for statistical analysis.

Results The average follow-up was 17.7 months. Postoperative Cobb angles were significantly corrected and anterior height ratios of fractured vertebrae were well restored in all three groups $(p<0.01)$. Back pain was efficiently relieved according to VAS score change $(p<0.01)$. There were significant differences in values of blood loss and post-operative VAS scores (at three months) between group A and group C $(p<0.01)$. No significant difference concerning post-operative anterior height ratios of fractured vertebrae, Cobb angles and
\end{abstract}

Jianhua Lyu and Kai Chen contributed to the work equally and should be regarded as co-first authors

Qiulin Zhang

zhangqiulin@189.cn

1 Department of Orthopaedics, The 92 Hospital of the People's Liberation Army, Nanping, Fujian Province, China

2 Department of Orthopaedics, Changhai Hospital, Second Military Medical University, Shanghai, China correction losses was observed between group A and group B ( $p=0.580,0.840,0.215$, respectively).

Conclusion Percutaneous fixation not only provides the same reduction effect as open fixation, but also has an advantage of causing less operation related trauma which is beneficial to post-operative rehabilitation. The efficacy of three-level percutaneous fixation and two-level percutaneous fixation in the treatment of type A thoracolumbar fractures is not significantly different.

Keywords Thoracolumbar fracture $\cdot$ Percutaneous pedicle screw $\cdot$ Minimally invasive surgery (MIS) $\cdot$ Randomized controlled trial (RCT)

\section{Introduction}

Thoracolumbar fractures are the most common spinal injuries. It is controversial to treat those patients who are without neurological deficits with surgery. Nevertheless, researches have shown that clinical and radiological outcomes of patients who accepted operations were more favorable than those treated without surgical intervention [1]. The surgical treatment via posterior approaches, known as traditional open surgery, was popular for decades. In recent years, percutaneous pedicle screw fixation technique has been widely used $[2,3]$. The use of additional screws in the intermediate pedicles is also disputable. In order to compare the efficacy of different surgical treatments, a randomized controlled trial was conducted between September 2012 and January 2015 in the department of Orthopaedics, Changhai Hospital, Second Military Medical University, Shanghai, China. 
Table 1 Baseline demographic characteristics of the three groups

\begin{tabular}{|c|c|c|c|c|c|c|c|c|c|c|}
\hline \multirow[t]{2}{*}{ Group } & \multirow[t]{2}{*}{ Age (years) } & \multicolumn{2}{|c|}{ Gender } & \multicolumn{4}{|c|}{ Fractured site } & \multicolumn{3}{|c|}{ AO classification } \\
\hline & & Male & Female & $\mathrm{T} 11$ & $\mathrm{~T} 12$ & L1 & L2 & A1 & A2 & A3 \\
\hline A & $45.8 \pm 7.8$ & 15 & 15 & 3 & 10 & 14 & 3 & 15 & 4 & 11 \\
\hline B & $45.3 \pm 8.3$ & 16 & 14 & 4 & 11 & 12 & 3 & 19 & 5 & 6 \\
\hline $\mathrm{C}$ & $43.7 \pm 8.8$ & 12 & 18 & 2 & 9 & 14 & 5 & 17 & 5 & 8 \\
\hline$p$-value & 0.58 & 0.561 & & 0.938 & & & & 0.71 & & \\
\hline
\end{tabular}

\section{Materials and methods}

Between September 2012 and January 2015, a total of 104 consecutive patients were diagnosed as type A thoracolumbar fractures. Among them, 90 cases who had given informed consent were selected according to the following criteria. The inclusion criteria for this study were as follows: age 18-60 years, single-level fresh thoracolumbar fracture (within two weeks), no neurological deficit. Exclusion criteria were Type A1.1 fractures, pedicle fracture, osteoporotic or pathologic fracture, previous spinal trauma or disease, other major organ system or musculoskeletal injuries, pregnancy, and female patients who were in the menstrual period. According to $\mathrm{AO}$ classification, there were 51 A1 cases, 14 A2 cases and 25 A3 cases among them. Ninety patients were randomly assigned to three groups of 30 cases each. Patients in group A were treated with three-level percutaneous fixation. Patients in group B underwent two-level percutaneous fixation. And patients in group $\mathrm{C}$ accepted three-level open fixation via traditional posterior approaches. There was no statistically significant difference in age, gender, fractured site or types of AO classification among the three groups $(p>0.05)$ (Table 1).

Plain X-radiographs, computed tomography (CT) scans and magnetic resonance imaging (MRI) were obtained in order to determine the type of fracture, the compression ratio of spinal canal, whether there was an injury of posterior ligamentous complex (PLC), etc. [4]. Thoracolumbar injury classification and severity scores (TLICS) [5] of all cases were calculated. Each patient received plain X-radiographs two days after surgery. CT scans were also a necessity if there had been obvious compression of spinal canal before surgery.
The procedures followed were in accordance with the ethical standards of the responsible committee on human experimentation (institutional and national) and with the Helsinki Declaration of 1975, as revised in 2000.

\section{Description of percutaneous fixation surgery}

A single shot of antibiotics was administered pre-operatively. Under general anaesthesia with endotracheal intubation, each patient was positioned prone on a Jackson operating table. Chest and pelvis were supported by gel pads fixed on the table. Postural reduction was performed before surgery. Lateral fluoroscopy was taken with the purpose of determining the efficacy of postural reduction. Posteroanterior (PA) fluoroscopy was used for locating one level above and below the injured vertebra. The corresponding positions of pedicles were marked on the patient's back. After undergoing disinfection of the surgical area, skin incisions were made $1.5-\mathrm{cm}$ lateral from the marks of those pedicles. Underlying fascia was cut by an electrosurgical knife and paraspinal muscles were split by a finger. Under PA fluoroscopy, each pedicle was cannulated by a Jamshidi needle at the lateral and medial margin. The insertion directions were adjusted according to lateral fluoroscopy. The needle was then slowly implanted through a pedicle. Via PA view it was made sure that each needle was not passed across the medial border of the corresponding pedicle, which suggested that there was no violation of spinal canal. A guidewire was placed into the vertebral body through the needle, followed by sequential dilators which could protect paraspinal muscles while creating a space for tapping. The dilators were then removed, allowing a percutaneous
Fig. 1 Images from a 35-year-old female in group A. a, b Preoperative radiographs indicating a fracture of T12. $\mathbf{c}, \mathbf{d}$ Post-operative radiographs indicating favorable restoration of anterior height of $\mathrm{T} 12$ vertebra and correction of Cobb angle
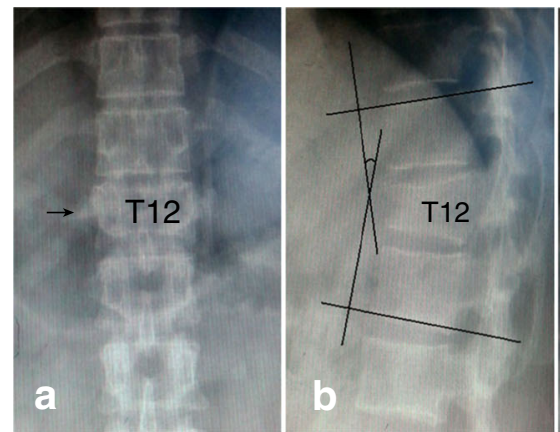

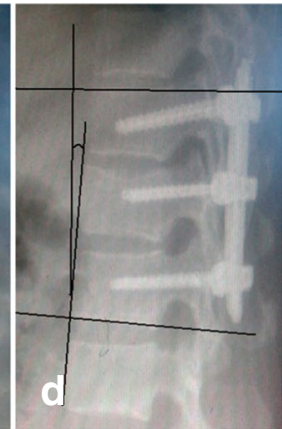


Fig. 2 Images from a 43-year-old male in group B. a, b Pre-operative radiographs indicated a fracture of L1. c, d Post-operative radiographs indicated favourable restoration of anterior height of L1 vertebra and correction of Cobb angle
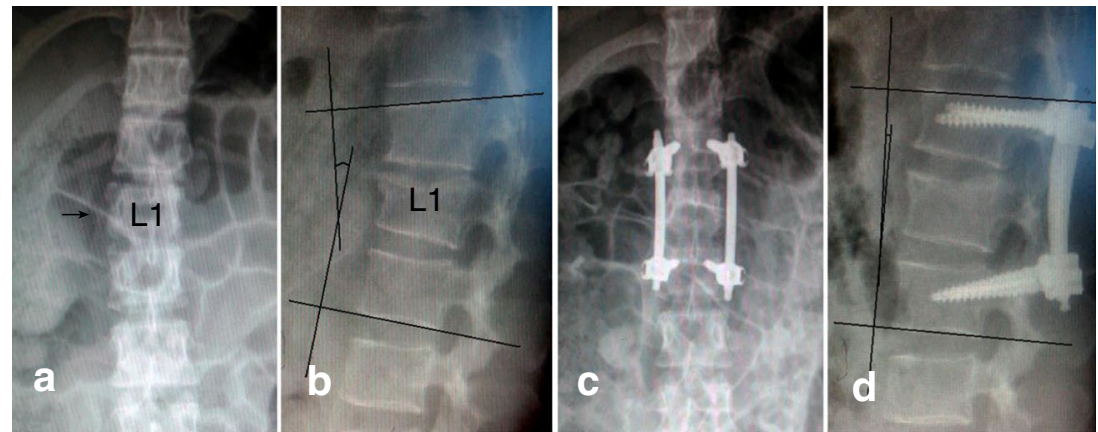

pedicle screw to be inserted into the pedicle and vertebral body along the wire which was also pulled out soon after. Each percutaneous pedicle screw was inserted into a pedicle and a vertebral body in the same way. The adjacent levels of fractured vertebra were implanted with bilateral pedicle screws in group B while an additional pair of screws were implanted into the fractured vertebra in group A. Therefore, there were six percutaneous pedicle screws implanted into each patient's thoracolumbar region in group A (Fig. 1d, e) and four screws in group B (Fig. 2d, e). The length of rods was assessed according to the distance between cranial and caudal screws. An appropriately bent rod was placed in the screws' heads of one side below the fascia. The height of fractured vertebra was further restored by applying a hyperlordosing force through the posterior elements just before the set of screws were tightened. The same procedure was followed on the other side. Good location of internal fixation and satisfactory correction of kyphosis were confirmed by PA and lateral fluoroscopy. Finally, incisions were irrigated and closed layer-by-layer without drainage.

\section{Description of open fixation surgery}

Dose-appropriate antibiotics were also administered at the induction. General anaesthesia and endotracheal intubation were conducted in the same way in group A and group B. Postural reduction and skin disinfection were also performed in a prone position for patients in group $\mathrm{C}$ on a Jackson operating table before the surgical procedure. After posterior thoracolumbar elements were clearly exposed through the traditional posterior approaches, the fractured and adjacent vertebrae were implanted with a total of six pedicle screws by free hands. Appropriately sized rods were placed in screws' heads and kyphosis was corrected by a hyperlordosing force. Subsequently, locking screws were tightened (Fig. 3d, e). Irrigation of the incision was repeatedly executed and drainage was placed before those layers were strictly closed. Generally, drainage was removed within 48 hours after the surgical procedure.

In the pre- and post-operative period, all patients were encouraged to do muscle flexing of lower limbs with the purpose of avoiding muscle atrophy and reducing the risk of deep venous thrombosis. Each patient in group A and group B was encouraged to have ambulatory activities while wearing a thoraco-lumbar-sacral brace three days after surgery. The starting time of ambulatory activities for patients in group $\mathrm{C}$ was two weeks post-operatively. All patients commenced back muscle exercises a fortnight after surgery. The brace was kept until three-month follow-up. Neither sports nor strenuous labour was allowed within six months after surgery. Plain X-radiographs were obtained at one month, three months, six months, one year and two years post-operatively. All patients accepted surgery of instrumentation removal during the period of six months to one year when plain $\mathrm{X}$-radiographs confirmed good healing of fractured vertebra.

The following radiological data were collected. Anterior height ratios (AHR) of fractured vertebrae and Cobb angles (CA) (Fig. 4) were measured in pre- and postoperative plain $\mathrm{X}$-radiographs (Figs. 1, 2 and 3b, e). The anterior height ratio
Fig. 3 Image from a 38-year-old male in group C. a, b Pre-operative radiographs indicated a fracture of L2. c, $\mathbf{d}$ Post-operative radiographs indicated favourable restoration of anterior height of L2 vertebra and correction of Cobb angle
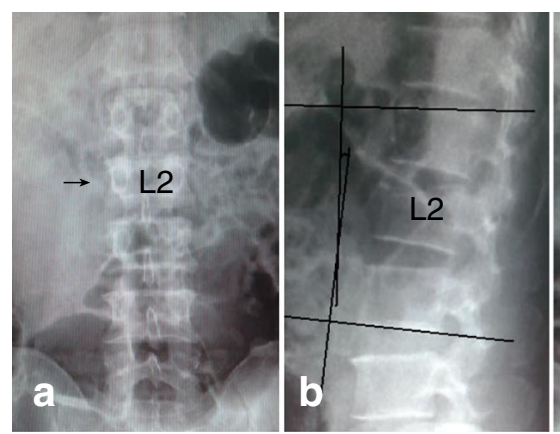

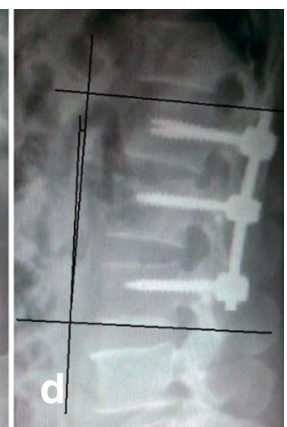




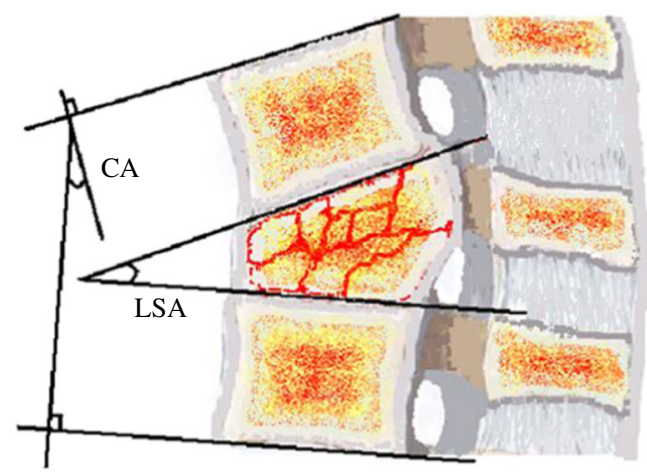

Fig. 4 Cobb angle (CA) and local sagittal angle (LSA)

of a fractured vertebra was calculated by dividing the anterior height of the fractured vertebra by its normal height. And the normal height of a fractured vertebra was estimated by taking the average between the anterior heights of upper and lower adjacent vertebrae. A Cobb angle was measured between the superior endplate of the upper vertebra and the inferior endplate of the lower vertebra. A correction loss was calculated by subtracting the Cobb angle of final follow-up from the first post-operative one.

Clinical parameters consisted of duration of surgery, intraoperative blood loss and visual analogue scale (VAS). VAS scores, assessing the magnitudes of patients' back pain, were measured at pre-operative time, three months follow-up and the final follow-up.

\section{Statistical analysis methods}

All radiological and clinical data were analyzed by using SPSS statistical package, version 16.0 (SPSS, Chicago, IL, USA). Chi-square test was used for analyzing categorical data among three different groups. Continuous variables were recorded as the mean $\pm \mathrm{SD}$. Among-group comparisons were made using ANOVA. If there was statistical significance, between-group comparisons were made by performing Dunnett- $t$ test. For each analysis, a $P$ value of $<0.05$ was considered statistically significant.

\section{Results}

The average follow-up was 17.7 months (range 1125 months). There was no significant difference in baseline demographic characteristics among three groups $(p>0.05)$ (Table 1). No significant among-group difference concerning pre-operative VAS score, Cobb angle or anterior height ratio of fractured vertebra was observed $(p>0.05)$. No neurological complication was noted. One case in group A who had been diagnosed as diabetes suffered from post-operative wound infection four days after surgery. The infection was soon controlled after treatments, including irrigation, debridement and
Table 2 Clinical parameters (group A vs group C)

\begin{tabular}{llll}
\hline Parameter & Group A & Group C & $p$-value \\
\hline Duration of surgery & $72.1 \pm 12.5$ & $77.8 \pm 8.2$ & 0.068 \\
Blood loss & $100.7 \pm 18.9$ & $202.1 \pm 42.0$ & $<0.001$ \\
VAS at three months & $2.2 \pm 0.5$ & $3.3 \pm 0.5$ & $<0.001$ \\
\hline
\end{tabular}

rational use of antibiotics, without the removal of internal fixation. Screw loosening was observed in one case in group $\mathrm{B}$ at three months with no clinical symptom. The patient was suggested to reduce ambulatory activities, increase back muscle exercises and keep wearing a brace until the time when good healing of the fracture was confirmed. The instrumentation of the patient was removed six months after the first surgery, and the outcome was favourable at the final followup.

The average durations of surgery in group A, group B and group $\mathrm{C}$ were $72.1 \pm 12.5 \mathrm{~min}, 64.4 \pm 9.8 \mathrm{~min}$ and 77.8 $\pm 8.2 \mathrm{~min}$; and the values of intra-operative blood loss were $100.7 \pm 18.9 \mathrm{ml}, 89.1 \pm 17.3 \mathrm{ml}$ and $202.1 \pm 42.0 \mathrm{ml}$, respectively. Duration of surgery was significantly shorter in group $\mathrm{B}$ than group A $(p<0.05)$. There was no significant difference concerning duration of surgery between group A and group C $(p=0.068)$. The values of intra-operative blood loss in group A were significantly less than those in group $\mathrm{C}(p<0.05)$. VAS scores for back pain at three months after surgery were 2.2 $\pm 0.5,2.4 \pm 0.5$ and $3.3 \pm 0.5$ points in group A, group B and group $\mathrm{C}$. The scores in group A were significantly lower than those in group $\mathrm{C}(p<0.05)$. The difference between group A and B was not statistically significant $(p=0.612)$ (Table 2).

Post-operative Cobb angles were significantly corrected and anterior height ratios of fractured vertebrae were well restored in all three groups $(p<0.05)$ (Figs. 1, 2 and 3). However, correction loss, which was reflected in significant increases of Cobb angles, was observed in all three groups at the last follow-up $(p<0.05)$. The increases of Cobb angles in group A, B and C were $2.9 \pm 1.4^{\circ}, 3.7 \pm 2.2^{\circ}$ and $3.6 \pm 1.9^{\circ}$. There were no significant among-group differences in postoperative Cobb angles, anterior height ratios of fractured vertebrae or correction loss $(p>0.05)$ (Table 3$)$.

Table 3 Radiological parameters (group A vs group B)

\begin{tabular}{llll}
\hline Parameter & Group A & Group B & $p$-value \\
\hline Pre-operative CA & $16.7 \pm 5.5$ & $17.3 \pm 3.6$ & 0.717 \\
Post-operative CA & $5.6 \pm 2.5$ & $5.9 \pm 1.4$ & 0.840 \\
CA at the final follow-up & $8.6 \pm 2.1$ & $9.6 \pm 2.2$ & 0.162 \\
Correction loss & $2.9 \pm 1.4$ & $3.7 \pm 2.2$ & 0.215 \\
Pre-operative AHR & $65.1 \pm 9.3$ & $66.0 \pm 7.6$ & 0.381 \\
Post-operative AHR & $88.8 \pm 6.2$ & $89.5 \pm 3.7$ & 0.580 \\
\hline
\end{tabular}

$C A$ Cobb angle, $A H R$ anterior height ratio 


\section{Discussion}

The highest incidence of spinal fractures happened at the thoracolumbar region because of its special anatomical features [6]. It is considered that a vertebral height loss of more than $50 \%$, a local sagittal angle (LSA) (Fig. 4) of more than 20 degrees or posterior ligamentous complex (PLC) injury may be unstable findings for traumatic thoracolumbar fractures [7]. Type A thoracolumbar fractures are without PLC injury. Both conservative management and surgical intervention are advocated $[8,9]$. Surgical fixation provides not only better fracture reduction, but also initial stability which enables early mobilization. Thus, it can effectively decrease complications associated with prolonged recumbence [1, 7]. Although the outcomes of traditional open surgery for patients who had indications are generally favourable, the extensive damage of soft tissue unavoidably results in a relatively high incidence of failed back surgery syndromes $[10,11]$. With the rapid development of minimally invasive techniques for spine in recent years, percutaneous pedicle screw fixation has gained its popularity because the technique has an obvious advantage in protecting paraspinal muscles from iatrogenic denervation and atrophy. Lee et al. [12] pointed out that when compared with conventional posterior surgery, the current minimally invasive procedure had advantages of costing less surgical procedure-related trauma and ensuring earlier recovery. The results of this study also showed that the values of intra-operative blood loss in percutaneous fixation groups were significantly less than those in the open fixation group. Patients who underwent minimally invasive surgery had lower post-operative VAS scores at three-month follow-up, which indicates that back pain is properly associated with the damage of paraspinal muscles in the open surgical procedure. Besides, patients in the percutaneous fixation group are allowed to have ambulatory activities much earlier, and will be less exposed to complications of bed rest [13].

In the study, there was no significant difference concerning kyphosis correction between three-level percutaneous fixation and three-level open fixation. Although the current minimally invasive fixation technique has so many advantages, it is still at its early stage. Inevitably, disadvantages, including more exposure to radiation and higher degree of difficulty with fracture reduction cannot be ignored. However, such disadvantages are undoubtedly going to be reduced or even be avoided by the development of technology concerning spinal surgery navigation, robotic guidance and so on [14].

Even before the application of minimally percutaneous fixation procedure, the idea of placing pedicle screws at the level of the fractured vertebra, causing considerable controversy, was proposed by some authors. They considered the reduction procedure with short segment fixation across fractured vertebra, or named two-level fixation, to be indirect. Moreover, a higher proportion of instrumentation failure and a larger degree of correction loss were observed in patients with twolevel fixation. In 1994, Dick et al. [15] conducted a study which indicated that the use of additional screws in the intermediate pedicles increased axial, sagittal, and torsional stiffness by varying degrees. Therefore, the technique could probably decrease correction loss. Besides, the three-level fixation provides a better pull-out strength and thus theoretically reduces the rate of instrumentation failure. However, in this study, there was no significant difference in Cobb angle restoration and correction loss between two-level fixation and three-level fixation. The reasons may be as follows. Firstly, the sample size of the study was not big enough, which resulted in an error. Secondly, there had been no damage at posterior elements of thoracolumbar in all patients, so the two-level fixation might be adequate for fracture reduction and stability maintenance. Thirdly, assisted by a thoraco-lumbar-sacral brace after surgery, the two-level fixation could sufficiently maintain the stability of the injured segment. Thus, it is not necessary to treat every case of thoracolumbar fracture with intermediate screws. In spite of the result of this study, the conclusion that the three-level fixation increases the stiffness of the fractured segment cannot be refuted. The use of intermediate screws is recommended when a patient is with severe osteoporosis, obesity, obvious injury of posterior column, etc.

Furthermore, it is worth noting that no posterolateral bony fusion was performed either in percutaneous surgery or in the traditional open procedure in the study, as several researches proved that posterior pedicle instrumentation without fusion could get comparable results as fixation with fusion [16].

There are two main limitations in the study. First, the study failed to use sagittal index (SI) to describe sagittal deformity, which influenced the accuracy of the results to some extent. Second, a few cases were not followed up long enough. Thus, long-term clinical outcomes of these cases, for instance, correction loss after the removal of instrumentation, could not be observed.

Although there are some deficiencies in this study, the results still show that the outcomes of both percutaneous and open surgery are satisfactory. Compared with the open fixation surgery, the percutaneous fixation surgery has an advantage of causing less operation-related trauma which is beneficial to postoperative rehabilitation. The efficacy of three-level percutaneous fixation and two-level percutaneous fixation in the treatment of type A thoracolumbar fractures is not significantly different.

Acknowledgments I would like to express my gratitude to all those who helped us during the writing of this thesis. I gratefully acknowledge the help of my supervisor, Professor Zhang Qiulin, who has offered me valuable suggestions in the academic studies. In the preparation of the thesis, he has spent much time reading through each draft and provided me with inspiring advice. Without his patient instruction, insightful criticism and expert guidance, the completion of this thesis would not have been possible. 


\section{Compliance with ethical standards}

Funding No external funding sources were used in the completion of this project.

Conflicts of interest The authors have no competing interests to declare.

Open Access This article is distributed under the terms of the Creative Commons Attribution 4.0 International License (http:// creativecommons.org/licenses/by/4.0/), which permits unrestricted use, distribution, and reproduction in any medium, provided you give appropriate credit to the original author(s) and the source, provide a link to the Creative Commons license, and indicate if changes were made.

\section{References}

1. Siebenga J, Leferink VJ, Segers MJ et al (2006) Treatment of traumatic thoracolumbar spine fractures: a multicenter prospective randomized study of operative versus nonsurgical treatment. Spine (Phila Pa 1976) 31:2881-2890

2. Proietti L, Scaramuzzo L, Schirò GR et al (2014) Posterior percutaneous reduction and fixation of thoraco-lumbar burst fractures. Orthop Traumatol Surg Res 100(5):455-460

3. Palmisani M, Gasbarrini A, Brodano GB et al (2009) Minimally invasive percutaneous fixation in the treatment of thoracic and lumbar spine fractures. Eur Spine J 18(Suppl 1):71-74

4. Crosby CG, Even JL, Song Y et al (2011) Diagnostic abilities of magnetic resonance imaging in traumatic injury to the posterior ligamentous complex: the effect of years in training. Spine J 11: 747-753

5. Vaccaro AR, Zeiller SC, Hulbert RJ et al (2005) The thoracolumbar injury severity score: a proposed treatment algorithm. J Spinal Disord Tech 18:209-215
6. Dai LY, Yao WF, Cui YM, Zhou Q (2004) Thoracolumbar fractures in patients with multiple injuries: diagnosis and treatment-a review of 147 cases. J Trauma 56:348-355

7. Butt MF, Farooq M, Mir B et al (2007) Management of unstable thoracolumbar spinal injuries by posterior short segment spinal fixation. Int Orthop 31(2):259-264

8. Mehmet T, Erden Erturer R, Ozturk C et al (2005) Conservative treatment of fractures of the thoracolumbar spine. Int Orthop 29(2): 78-82

9. Verlaan JJ, Diekerhof CH, Buskens E et al (2004) Surgical treatment of traumatic fractures of thethoracic and lumbar spine: a systematic review of the literature on techniques, complications, and outcome. Spine (Phila Pa 1976) 29:803-814

10. Sihvonen T, Herno A, Paljarvi L et al (1993) Local denervation atrophy of paraspinal muscles in postoperative failed back syndrome. Spine 18:575-581

11. Fan S, Hu Z, Zhao F et al (2010) Multifidus muscle changes and clinical effects of one-level posterior lumbar interbody fusion: minimally invasive procedure versus conventional open approaches. Eur Spine J 19(2):316-324

12. Lee JK, Jang JW, Kim TW et al (2013) Percutaneous short-segment pedicle screw placement without fusion in the treatment of thoracolumbar burstfracture: is it effective?: comparative study with open short-segment pedicle screw fixation with posterolateralfusion. Acta Neurochir (Wien) 155(12):305-2312

13. Kim DY, Lee SH, Chung SK, Lee HY (2005) Comparison of multifidus muscle atrophy and trunk extension muscle strength: percutaneous versus open pedical screw fixation. Spine (Phila Pa 1976) 30:123-129

14. Lekovic GP, Potts EA, Karahalios DG et al (2007) A comparison of two techniques in image-guided thoracic pedicle screw placement: a retrospective study of 37 patients and 277 pedicle screws. J Neurosurg Spine 7:393-398

15. Dick JC, Jones MP, Zdeblick TA et al (1994) A biomechanical comparison evaluating the use of intermediate screws and crosslinkage in lumbar pedicle fixation. J Spinal Disord 7(5):402-407

16. Dai LY, Jiang LS, Jiang SD (2009) Posterior short-segment fixation with or without fusion for thoracolumbar burst fractures. a five to seven-year prospective randomized study. J Bone Joint Surg Am 91:1033-1041 